\title{
Image Processing and Analysis of Textile Fibers by Virtual Random Walk
}

\author{
Hafedh Zghidi, \\ Maksym Walczak \\ Silesian University of Technology, \\ Faculty of Automatic Control, \\ Electronics and Computer Science, \\ 44-100 Poland \\ E-mail: hafed.zghidi@polsl.pl, \\ maksym.walczak@gmail.com
}

\author{
Tomasz Blachowicz, \\ Krzysztof Domino \\ Silesian University of Technology, \\ Institute of Physics - Center for \\ Science and Education, 44-100 \\ Poland \\ E-mail: \\ tomasz.blachowicz@polsl.pl, \\ krzysztof.domino@polsl.pol
}

\author{
Andrea Ehrmann \\ Bielefeld University of Applied \\ Sciences, Faculty of Engineering \\ Sciences and Mathematics, 33609 \\ Germany \\ E-Mail: andrea.ehrmann@fh- \\ bielefeld.de
}

\begin{abstract}
An algorithm for extracting material shape and spatial information from non-uniform background, and for generating object skeletons for statistical two-dimensional experiments using random walk approach, is presented. This finds applications in textile analysis and microscopic analysis of various materials like hairs and allows for further precise determination of textile yarn dimensions as well as other geometrical characteristics like a fractal dimension.
\end{abstract}

\section{INTRODUCTION}

$\mathrm{H}^{2}$ EMP fibers can be used for a variety of textile products, e.g. clothing or home textiles, but also for technical purposes in fiber composites. For all applications, it is important to determine the fiber lengths and diameters (averaged along with a standard deviation), a task which can be handled by optical equipment and respective software (e.g. [1,2]). Another important factor, however, is the identification of the degree of bifurcation, which influences the spinning process, the fiber-matrix bond strength etc. For this property there is no standard measurement procedure available. Our article aims at presenting a novel method to define the bifurcation of fibers, based on the evaluation of microscopic fiber images based on random walk analysis. The hemp fibers investigated in our study are pre-treated by hammer milling or by combing. Afterwards, the fibers are separated chemically by alkaline cooking [3].

Photographic images of di erent magni cations were taken using a digital optical microscope VHX-600D by Keyence with an objective VH-Z20R. Captured images were then processed giving monochromatic representative maps. Next, random walk statistical experiments on these maps were carried out. Subsequent chapters describe all mentioned steps, before conclusions are nally provided.

\section{IMAGE PROCESSING}

The algorithm described here consists of two denoising and four extraction steps. In the first step, the image is being smoothed using a median filter [4]. This step slightly reduces fine-grained noise and also improves the efficiency of filters used in the first extraction step of the algorithm. The next step is an unsharp masking where it is necessary to define the proportional factor $\mathrm{k}$ of the operation [5]. This step can be defined as follows:

$$
\begin{gathered}
A_{u m}=A_{m f}-\text { Gauss }\left(A_{m f}\right), \\
A_{u m}=k \cdot A_{u m}+A_{m f},
\end{gathered}
$$

where $A_{u m}$ is the unsharp masked image, $A_{m f}$ is the median filtered image, and Gauss is the Gaussian filter [4], for which we use the Gaussian filter kernel $k=0.4$. The window size and the standard deviation parameter $\sigma$ equal 3 . The parameters were chosen experimentally.

The first extraction step is an algorithm that can be split into two functional parts: a background sampling, and a background removal. The algorithm removes the background using a special filter that uses a background sample. The filter window is defined as a vector $h=\left\{h_{1}, h_{2}\right.$, $\left.h_{3}, h_{4}\right\}$, where $h_{1}$ is the average intensity, $h_{2}$ is the standard deviation of intensity, $h_{3}$ is the maximum intensity, and $h_{4}$ the minimum intensity. Contrary to the results of Ref. 6, all the values from $h_{1}$ to $h_{4}$ are calculated from a Moore neighborhood of each pixel. This improves the filter's quality as consecutive neighborhoods partially overlap each other.

In the first functional part, the algorithm sums Euclidean distances from reference vector, created from upper left corner of the background sample $\left(h_{r e f}\right)$, to reference vectors of all the other pixels from the sample $\left(h_{i b}\right)$. Next, the algorithm calculates the reference pattern $\bar{d}$ that can be expressed as follows

$$
\bar{d}=\frac{\sum_{i=0}^{i=M \cdot N} d\left(h_{r e f}, h_{i b}\right)}{s},
$$

where $s$ is the experimentally chosen value, $M$ is the number of rows in the background sample, and $N$ is the number of columns in the background sample.

There are two options for choosing the $s$ value. The default value is $1 / 4 M N$, which makes $\bar{d}$ an average of all the distances, taking into consideration the window size $=3$. 
The other option is to divide by an experimentally chosen value to either increase or decrease the algorithm's sensitivity. For the pictures presented in this article we chose the second option. Thus, it was necessary to divide the sum by a number smaller than $1 / 4 M N$. This choice increased the filter's sensitivity, since the background in our images was far less uniform than in the images in [6]. Once the $s$ value is properly chosen, the algorithm will give similarly good results for other images provided their background has the same texture and the lighting conditions are similar. This procedure will be proved in the next section of this paper.

In the second part of the algorithm, the distance between reference vectors, generated for each pixel of the image, is compared to the previously calculated reference pattern. The filter function is defined as

$$
\begin{gathered}
A_{d n} \text { if } d\left(h_{r e f}, h_{i}\right) \geq \bar{d} \\
0 \text { if } d\left(h_{r e f}, h_{i}\right)<\bar{d},
\end{gathered}
$$

where $h_{\text {ref }}$ is the reference vector created from the upper left corner of the sampled region, $h_{i}$ is the $i$-th region of the whole image, and $A_{d n}$ is the denoised image. When the distance is greater than the reference pattern, we leave the processed image untouched, otherwise we remove the currently sampled pixel. Eventually any objects in the image are separated from the background.

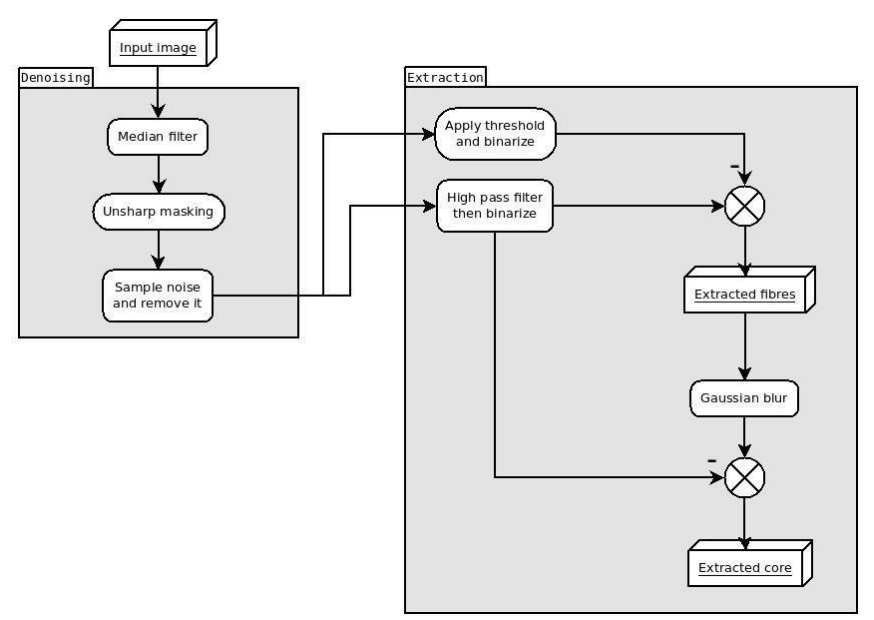

Fig. 1 Pipeline of the algorithm

The algorithm requires several parameters to be specified in order to process input images with expected quality. These parameters are: the background sample, the extraction threshold, the sensitivity, the median filter size, the unsharpmasking proportional-factor, the Gaussian filter window size, and the Gaussian and high-pass filter window-size. For the purpose of this paper, however, we analyzed all the parameters and chose to present only those which have the greatest impact on image quality. The results comparing influence of parameters for the same input image are presented in Fig. 2. In Fig. 3 only input images and final results needed for random walk experiments are depicted.

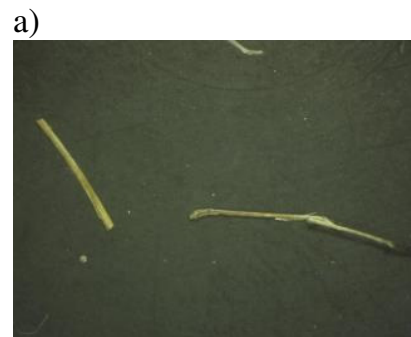

c)
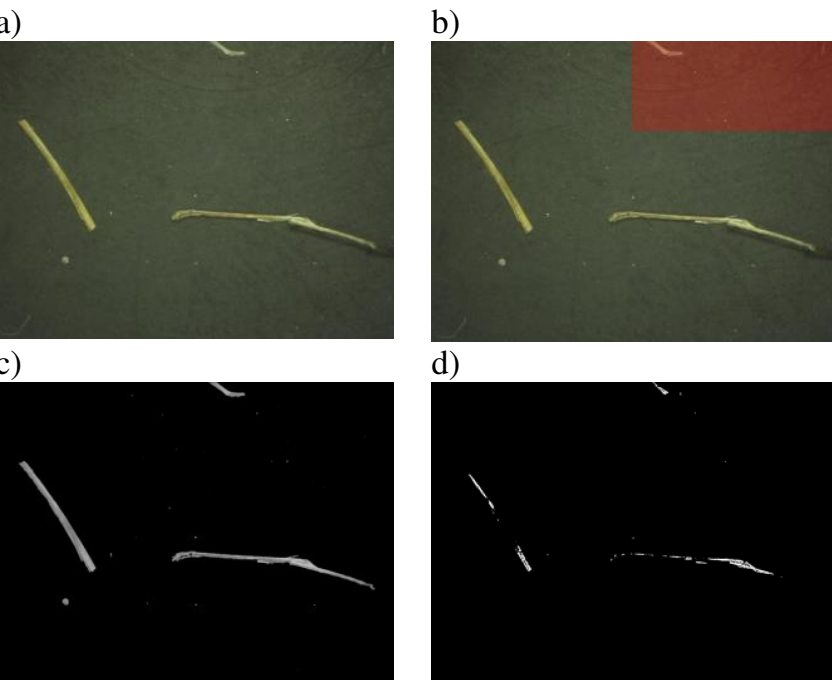

e)
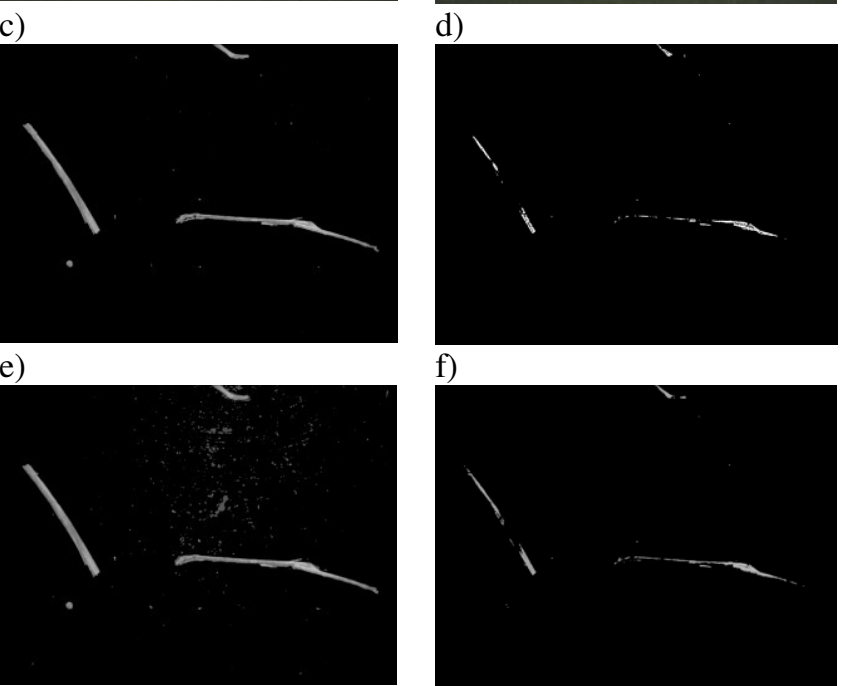

f)

Fig. 2 A first exemplary set with optimally chosen parameters (segmentation threshold $=100, s=0.06 \cdot$ width $\cdot$ height): input image for processing (a), background sample (b), image separated from background (c), second result set for image separated from the background with too high sensitivity, the same background sample as above (segmentation threshold $=100, \mathrm{~s}=0.05 \cdot$ width $\cdot$ height) (d), third set of results for the image separated from the background with too low sensitivity, the same background sample as above (segmentation threshold $=100, s=0.07 \cdot$ width $\cdot$ height) (e), a fourth set of results for the image separated from the background with bad noise sample (segmentation threshold $=100, s=0.06$. width $\cdot$ height) (f).

As it is seen in the examples, the presented parameters have very high impact on the image quality. Although the parameter $s$ needs to be chosen experimentally, different input images having similar background texture and lighting conditions are being processed properly. This proves the algorithm's applicability in microscopic photography and industrial photography, where different objects are photographed in similar, but not necessarily identical conditions. 
a)

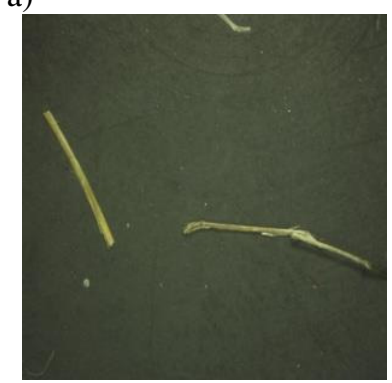

c)

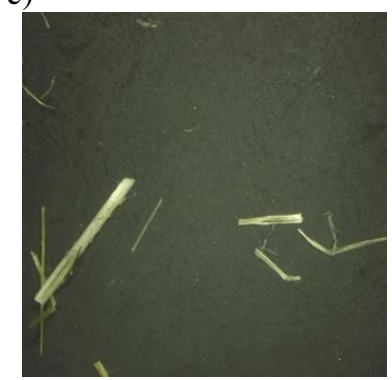

e)

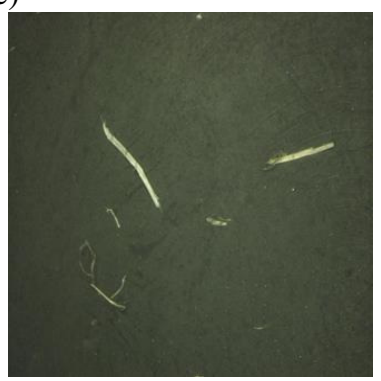

b)

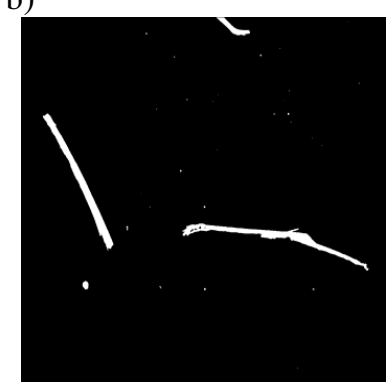

d)

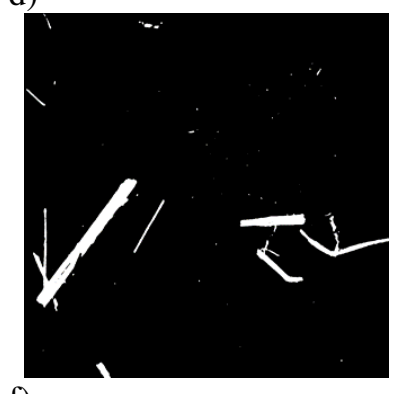

f)

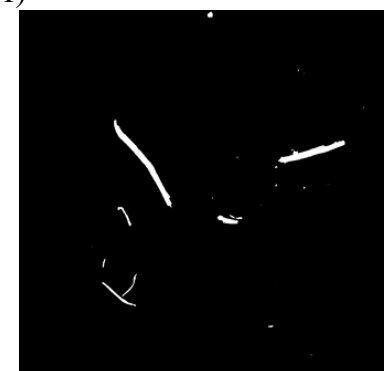

Fig. 3 Different examples of input images (a, c, e) and final results of monochrome maps needed for random walk experiments $(b, d$, f), respectively

\section{RANDOM WALK EXPERIMENTS}

In this chapter the use of the image statistical analysis to examine the time series of the displacement of the random walker is discussed. Let us consider a uniform twodimensional 'black' plane. A random walk can be applied and a walker is allowed to move randomly (with the same probability) up, down, to the left or to the right by a unit length in a unit time. This is the Markovian type random walk and the direction of the $n$-th step is chosen at random and does not depend on directions of previous ones. Here the mean square displacement of the probe $\left\langle R^{2}(n)\right\rangle$ is linearly dependent on the number of steps $n$ (time) which is proportional to the squared averaged random walk distance:

$$
\left\langle R^{2}(n)\right\rangle \propto n \text {. }
$$

Similarly, the pictures under investigation can be examined by means of the random walk procedure. At every iteration, a random direction is being chosen and then the algorithm proceeds one step into the chosen direction. However, the position at the $n$-th step depends on the previous positions due to the non-uniformity of the texture. Finally, the mean

squared displacement is no longer the linear function of $n$ [79], but the following relation appears for large $\mathrm{n}$ :

$$
\left\langle R^{2}(n)\right\rangle \propto n^{2 H},
$$

where $H$ is the self-similarity coefficient called Hurst exponent [7,10]. From Hurst exponent distribution, unambiguous information about yarn hairness, and in general, information about yarn quality can be obtained. Such quantitative evaluations are on demand for textile industry. In practice, for a random walk on two-dimensional monochromatic pictures the typical result is $H<1 / 2$; however, the information obtained for the exponent distribution is a unique information of a given picture (Fig. 4). Details about this type of analysis, applied for knitted fabrics, can be found in [11].

a)

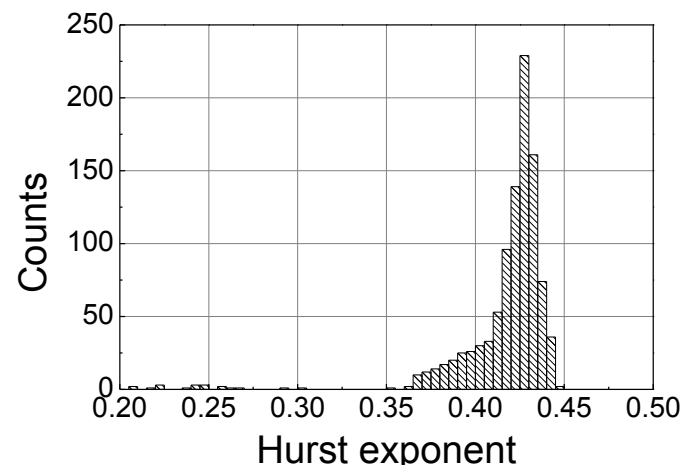

b)

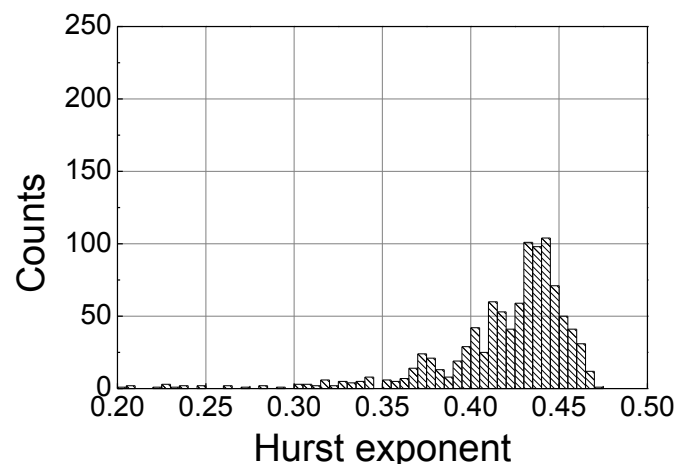

c)

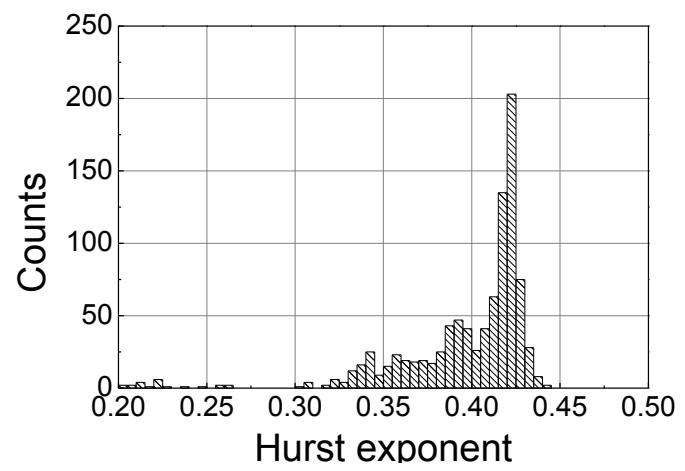

Fig. 4 Results of random walk experiments (a, b, c) carried out on the three monochromatic images from Fig. 3 (b, d, f), respectively 


\section{CONCLUSIONS}

The paper gives an overview of the necessary steps used to create reliable monochromatic images from microscopic images by separating objects under examination from the background. This algorithm was applied on microscopic pictures of hemp fibers. Comparison between random walks performed on the monochromatic pictures gives a quantitative measure of differences between the hemp fibers, dependent on the fiber diameters and bifurcations which are important parameters in fiber manufacturing processes such as spinning or composite production. Thus, the image processing algorithm presented here is a fundamental requirement in the optical evaluation of fiber quality using the self-similarity coefficient.

\section{REFERENCES}

[1] H. L. Bos, J. Müssig, and M. J. A. van den Oever, "Mechanical properties of short-flax-fibre reinforced compounds, Composites Part A," Applied Science and Manufacturing 37, pp. 1591-1604, Oct. 2006. DOI:10.1016/j.compositesa.2005.1-0.011

[2] J. L. Thomason, J. Carruthers, J. Kelly, and G. Johnson, "Fibre crosssection determination and variability in sisal and flax and its effects on fibre performance characterization," Composites Science and Technology 71, pp. 1008-1015, May 2011. DOI:10.1016/j.compscitech.2011.03.007

[3] A. Gutiérrez and J. C. del Rio, "Lipids from Flax Fibers and Their Fate in Alkaline Pulping," J. Agric. Food Chem. 51, pp. 4965-4971, Jul 2003. DOI: $10.1021 / \mathrm{jf} 034370 \mathrm{t}$

[4] Mark Nixon, "Feature Extraction \& Image Processing for Computer Vision", Academic Press, Third edition p. 109, 2012

[5] Mark Nixon, "Feature Extraction \& Image Processing for Computer Vision", Academic Press, Third edition p. 114, 2012

[6] A. Fabiańska, and L. Jackowska-Strumiłło, "Image processing and analysis algorithms for yarn hairiness determination," Machine Vision and Applications 23, pp. 527-540, Feb. 2012. DOI: 10.1007/s00138012-0411-y

[7] S. Havlin, and D. Ben-Avraham, "Diffusion in disordered media," Advances in Physics, 51, pp. 187-292, Jan. 2002. DOI: $10.1080 / 00018730110116353$

[8] J. W. Haus, and K. W. Kehr, "Diffusion in Regular and Disordered Lattices," Phys. Rep. 150 , pp. 263-406, Jun. 1987. DOI: 10.1016/03701573(87)90005-6

[9] D. Ben-Avraham, and S. Havlin, Difffusion and Reactions in Fractals and Disordered Systems, Cambridge Univ. Press, Cambridge 2000.

[10] S. Alexander, and S. R. Orbach, "Density of states on fractals: fractons," J. Phys. Lett., Paris, 43, pp. 625-631, Sept. 1982. DOI: 10.1051/jphyslet:019820043017062500

[11] A. Ehrmann, T. Blachowicz, K. Domino, S. Aumann, M. O. Weber, and H. Zghidi, "Examination of hairiness changes due to washing in knitted fabrics using a random walk approach," Textile Research Journal, online first, April 16, 2015. DOI: 10.1177/0040517515581591 